\title{
Design on Generating Test Paper Based on Simulated Annealing Algorithm
}

\author{
Jun Yang
}

\begin{abstract}
This paper introduces the simulated annealing algorithm to optimize test paper problem based on the standard genetic algorithm. Firstly, its mathematical model was established in the light of more constraints while generating test paper. In the calculation, these six attributes are needed to consider, they are the score, type, difficulty, knowledge, chapter and time; secondly, describing the simulated annealing algorithm; finally, the four management modules of test paper system were described in detail. In order to avoid the premature shortcoming of genetic algorithm, you can use simulated annealing algorithm to stretch individual papers. The algorithm has a higher significance in solving practical problems in learning activities, which is worth promoting the use.
\end{abstract}

Keywords-genetic algorithm, simulated annealing algorithm, mathematical model, premature.

\section{INTRODUCTION}

With the rapid development of computer and network technology, computer plays a more and more important role in the modern learning [1]. As a branch of computerassisted instruction, intelligent test paper is one of the key problems of computer-aided instruction. With its platform of modern computers, the advanced algorithms' theory and the experience of experts can be combined to develop a learning application platform of examination papers with expert level. By using this platform to reduce multiple procedures in the traditional exams, so that human, financial resources and material will be saved. Meanwhile, computers review papers to ensure the objectivity, and the fairness of the test scores can be improved. The quality of test paper is the key to evaluate test paper system. Currently, there are many algorithm used in the field of intelligent test paper, they are genetic algorithm and its improved algorithm, test paper generating algorithm based on granularity synthesis calculation, adaptive algorithm and particle swarm optimization and so on. The genetic algorithm with adaptive optimization and a good search feature has been favored by many scholars [2,3]. However, genetic algorithm has weak local search capability and is prone to produce precocious problem. In order to overcome the shortcoming of genetic algorithm to further improve the search efficiency of the algorithm, this paper introduces the simulated annealing algorithm based on the standard genetic algorithm to improve the performance of the algorithm.

\author{
School of Education and Sports, Bohai University, \\ Jinzhou, 121013, China
}

\section{ESTABLISHMENT OF MATHEMATICAL MODEL ON GENERATING TEST PAPER}

Whether a good intelligent test paper system can perform their functions to help teachers to complete the learning work better, mainly to see if it generates the papers have good reliability and validity, on the one hand, this depends on the quality of questions, on the other hand, it depends on the realization of the algorithm. The mathematical model of test paper problem is the basis of intelligent test paper, and its solution process is a process for solving multi-objective constraints to meet. That is, if you retrieve a set of questions in line with the test paper requirements from a number of item banks, so that the problem of automatic test paper becomes a problem with multiple constraints, you can define a set of constraints and a combination of objective function to describe the problem. According to the test paper requirements that are constraints, questions can be chose [4-7].

Suppose there is a paper in the test paper, actually it is a $p \times q$ target matrix. Each question includes the following attributes: score $\left(U_{1}\right)$, type $\left(U_{2}\right)$, difficulty $\left(U_{3}\right)$, knowledge $\left(U_{4}\right)$, chapter $\left(U_{5}\right)$, time $\left(U_{6}\right)$ and so on. Wherein, $p$ represents the number of questions included in the paper, and each question has $q$ attributes, namely the $q$ constraints. So you can use the matrix $E$ to represent. Among them, the rows say the questions, and the columns represent question' attributes. In this article, assume there are $p$ questions, and each question has 6 attributes, as described above. For example, $a_{i j}$ represents the $j$-th attribute in the $i$-th question, wherein, $i=1,2,3, \cdots p, \quad j=1,2,3, \cdots, 6$.

$$
E=\left[\begin{array}{cccc}
a_{11} & a_{12} & \cdots & a_{1 p} \\
a_{21} & a_{22} & \cdots & a_{2 p} \\
\vdots & \vdots & \ddots & \vdots \\
a_{q 1} & a_{q 2} & \cdots & a_{q p}
\end{array}\right]
$$

(1) Score constraint: $W=\sum_{i-1}^{p} a_{i 1}$, score $W$ is 100 .

(2) Type constraint: suppose the papers include following types of questions: multiple choice, fill in the blank and calculation problem. They constitute the second column of the target matrix $E$, the score of each type of questions is set by the user himself, with $W_{1 j}$ representation. The following conditions need to be satisfied: 


$$
\sum_{i=1}^{p} y_{j} \times x_{i, 2}=W_{1 j} \quad W_{1 j}= \begin{cases}1, & x_{i, 2}=j \\ 0 & x_{i, 2} \neq j\end{cases}
$$

(3) Difficulty constraint: suppose the papers include following types of difficulty: easy, medium, difficult, expand. They constitute the third column of the target matrix $E$, the score of each type of difficulty is set by the user himself, with $W_{2 j}$ representation. The following conditions need to be satisfied:

$$
\sum_{i=1}^{p} y_{j} \times x_{i, 3}=W_{2 j} \quad W_{2 j}= \begin{cases}1, & x_{i, 3}=j \\ 0 & x_{i, 3} \neq j\end{cases}
$$

(4) Knowledge constraint: you can set the proportion of each knowledge point and the score of this knowledge according syllabus. This attribute constitutes the fourth column of the target matrix $E$, the each knowledge score is set by the user himself, with $W_{3 j}$ representation. The following conditions need to be satisfied:

$$
\sum_{i=1}^{p} y_{j} \times x_{i, 4}=W_{3 j} \quad W_{3 j}= \begin{cases}1, & x_{i, 4}=j \\ 0 & x_{i, 4} \neq j\end{cases}
$$

(5) Chapter constraint: the number of questions and score in each chapter are calculated according to syllabus. This attribute constitutes the fifth column of the target matrix $E$, the score of questions in each chapter is set by the user himself, with $W_{4 j}$ representation. The following conditions need to be satisfied:

$$
\sum_{i=1}^{p} y_{j} \times x_{i, 5}=W_{4 j} \quad W_{4 j}= \begin{cases}1, & x_{i, 5}=j \\ 0 & x_{i, 5} \neq j\end{cases}
$$

(6) Time constraint: the total time is set by the user himself, with $T$ representation. The time to complete $i$-th question is $t_{i}$, so the time $T$ needs to satisfy the following conditions:

$$
T=\sum_{i=1}^{p} t_{i}
$$

\section{DESCRIPTION SIMULATED ANNEALING ALGORITHM}

Genetic simulated annealing algorithm is integrated the idea of simulated annealing into genetic algorithm. That is, you can adjust the value of the parameter under the control of parameter (temperature), until that the selected parameter value can make function reach the global minimum eventually [8]. Genetic algorithm in solving problems is likely to produce precocious phenomenon, which cannot quickly find a viable solution in the local, so the phenomenon that a feasible solution appears in different searches cannot be avoided. The introduction of the idea of simulated annealing in genetic algorithm can effectively avoid such drawback. Genetic simulated annealing algorithm start the search from a given initial solution, a new set of population can be generated, and using simulated annealing algorithm to annealing for individuals of new population, and new individuals produced by annealing operation can be regarded as the individuals of next group in the genetic algorithm. The flow chart of algorithm is shown in Fig. 1.

The basic steps are[9,10]:

(1) Initialization: the initial temperature is $T$ (sufficiently large), the initial solution state is $S$ (the start of iterative algorithm), the number of iterations for each $T$ is $L$;

(2) For $k=1, \cdots \cdots, L$ does step 3 to step 6;

(3) Generating new solution $S^{\prime}$;

(4) Calculating the increment $\Delta t^{\prime}=C\left(S^{\prime}\right)-C(S)$, $C(S)$, is evaluation function;

(5) If $\Delta t^{\prime}<0$ accepts $S^{\prime}$ as the new solution, otherwise using the probability of $\exp \left(-\Delta t^{\prime} / T\right)$ to accept $S^{\prime}$ as the new solution;

(6) If the termination condition is satisfied, the current solution should be output as the optimal solution, the program ends. Termination condition is that when several consecutive new solutions have not been accepted, the algorithm will end;

(7) $T$ reduces gradually, and $T->0$, then go to step 2 . 


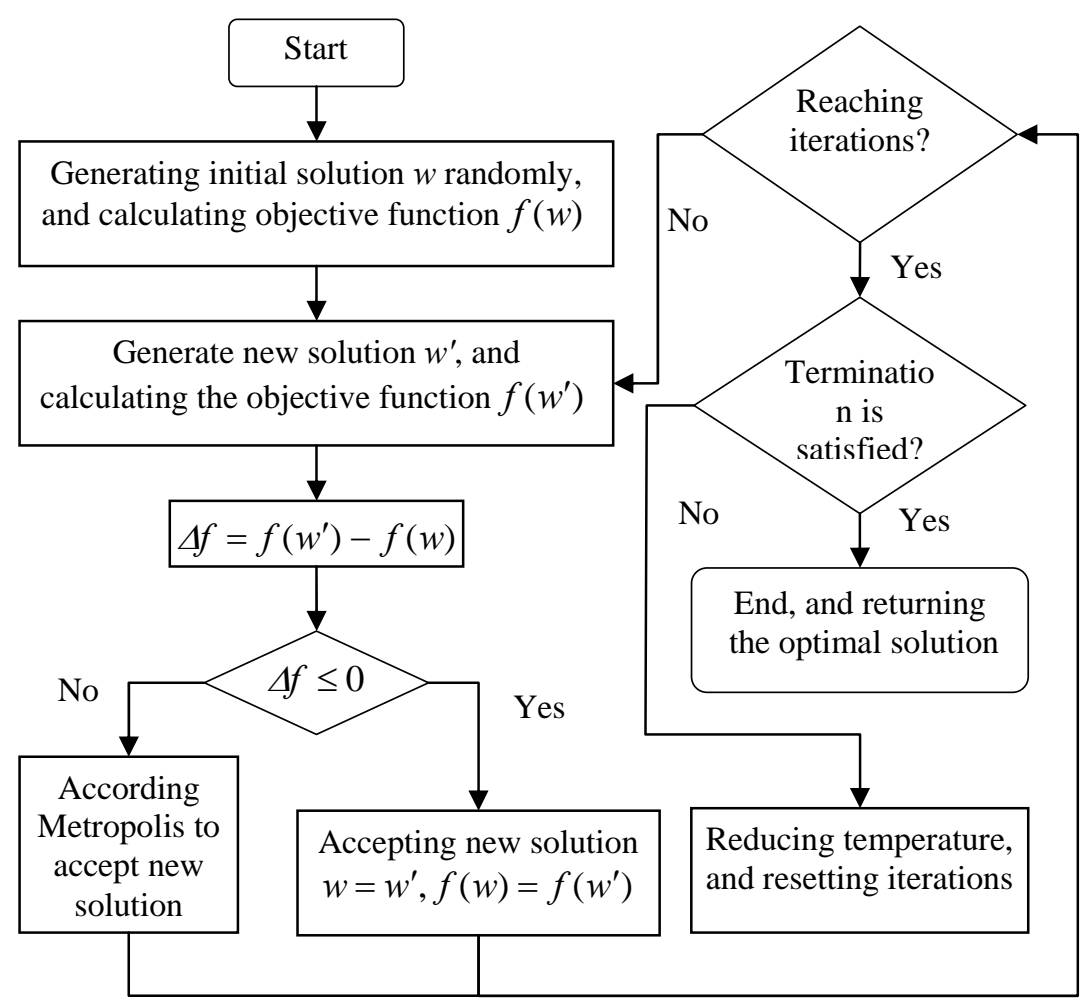

Figure 1. Flow chart of simulated annealing algorithm

\section{SYSTEM DESIGN}

To ensure the security of the system, you need to enter user name and password to enter the system. If you enter the correct user name and password, then you can enter the test paper system success, while the appropriate permission is given to the user; if the input information is incorrect, an error message will be appeared and you still remain in the landing page to continue until a successful landing [11]. In the process of automatic test paper, the module function can be achieved by performance management, question bank management, test paper management and paper management. Specific configuration is shown in Fig. 2. 


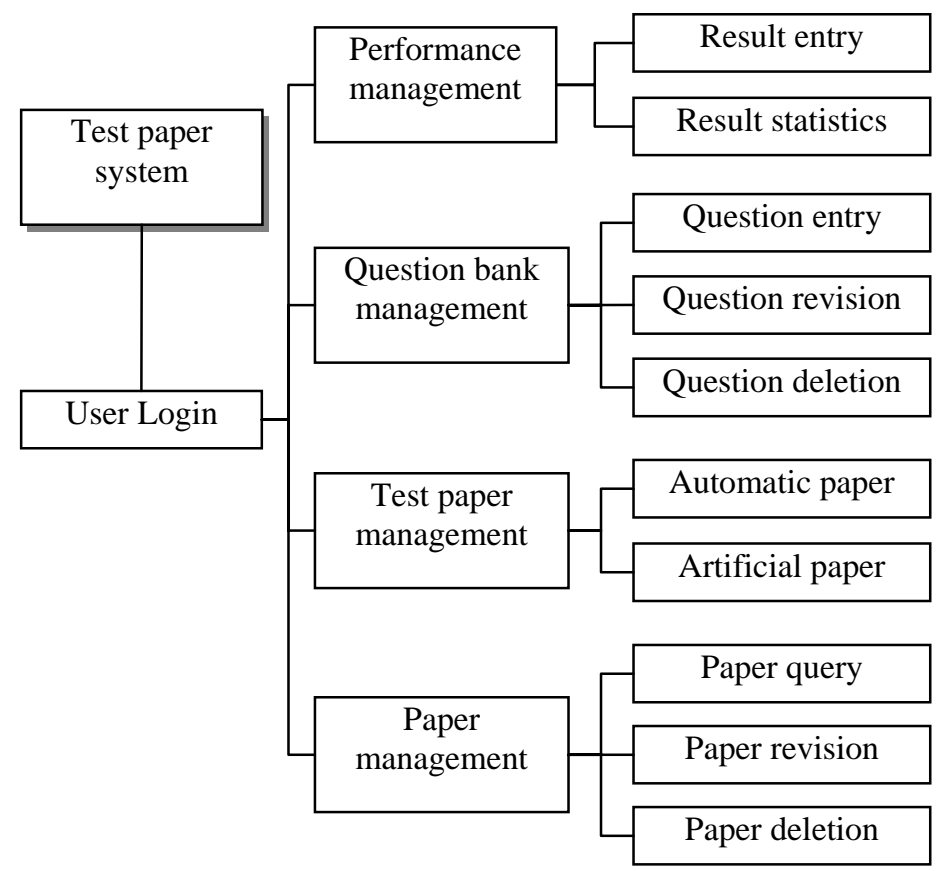

Figure 2. Functional structure chart of the system

Performance management module: after landing system, teachers can enter students' results, and results can be counted to sort;

Question bank management module: Paper system has questions in different modes in order to meet multidisciplinary courses, which involve multiple-choice, fill in the blank, and calculation question. Teachers who have permission can add, modify, or delete their own exam questions and performance indexes of the questions. When each kind of questions is entered, the basic attributes of type, difficulty, knowledge, chapter and time are set;

Test paper management module: the function of generating test paper is the critical of autogenerating test paper system. There are two kinds of test paper mode, namely are artificial test paper and automatic test paper;

Paper management module: after landing system, teachers can query generated papers previously; if not satisfied with the current generated papers, teachers can modify papers, or even empty generated papers.

\section{CONCLUSIONS}

The generation of test paper is a multi-objective optimization problem, which needs to take into account balance among the various constraints. Test paper is the key of intelligent examination system. This paper introduces the idea of simulated annealing algorithm due to that the genetic algorithm is easy to fall into local optimum and premature. The test paper system can get scientific and satisfied papers in a faster search speed due to use hybrid genetic algorithm [12]. The algorithm is applicable to all courses, the practice proves that the method can complete test paper work better, and the quality of test paper can better meet the distribution requirements of control targets. With the further popularization of paperless examination systems, the technology of intelligent test paper will increasingly become the focus of research.

\section{ACKNOWLEDGEMENTS}

The research work was supported by "12th Five Year Plan" of educational science planning project of Liaoning province No. JG13CB030, 2014 annual general undergraduate higher education reform project of Liaoning province (Research on action primary education professional training mode reform based on Standard), 2013 annual Bohai University teaching reform project No. JG13YB004.

\section{REFERENCES}

[1] Y. C. Zhou, Y. L. Liu, J. H. Gu. Research on Test Paper Autogenerating Based on Niche Adaptive Genetic Simulated Annealing Algorithms. Journal of Chinese Computer Systems, 32(2), pp. 324327, 2011.

[2] J. X. Zhao, J. H. Huang. Research of Intelligent Grouping Based on Genetic Simulated Annealing Algorithm. Computer Knowledge and Technology, 8(21), pp. 5183-5185, 2012.

[3] Baidu Baike. Simulated annealing algorithm. http://baike.baidu.com/link? url=u6CczkPMx3bM6Zl9lEJIiwUjgfg VJo-_HHD9Sh0kV7jAp0T0-dM2RB-LsN5_FpOEKjoZhrBmgcOp_UODuowua, 2015-01-21.

[4] Y. C. Zhou, Y. L. Liu. Research on auto-generating test paper based on genetic simulated annealing algorithms. Computer Engineering and Design, 32(3), pp. 1066-1069, 2011.

[5] J. Li. Research on Intelligent Test Paper Composition System Based on Genetic Algorithm. Master's degree of Tianjin University, 2008. 
[6] C. Y. Jiao. Research on Intelligent Test Paper Composition System Based on Genetic Algorithm. Master's degree of Nanjing University of Science \& Technology, 2011.

[7] J. F. Li, R. B. Lu, Z. S. Li. Research and implementation of intelligent test paper generation system, Computer \& Digital Engineering, 36(3), pp. 63-65, 2008.

[8] X. B. Lin. Research on a variety of common methods of automatic paper. Fujian Computer, 26(3), pp. 53-54, 2010.

[9] G. Q. Li, J. F. Yan. Research of intelligent test paper composition system based on genetic algorithm. Science \& Technology Information, 27(26), pp. 111-112, 2010.
[10] Lin Danping, Lee C.K.M., Wu Zhang. Integrating analytical hierarchy process to genetic algorithm for re-entrant flow shop scheduling problem. International Journal of Production Research, 50(7), pp. 1813-1824, 2012.

[11] F. Wang. Design and Implementation of Automatic Generating Test Paper System. Master's Degree of East China Normal University, 2010.

[12] S. Q. Yi, G. J. Wang. Research on Autogenerating Test Paper Based on Simulating Annealing Genetic Algorithm. Computer Engineering and Design, 29(17), pp. 4538-4540, 2008. 